\title{
Investigation of the Attitude Error Vector Reference Frame in the INS EKF
}

\author{
Stephen Steffes, Jan Philipp Steinbach, and Stephan Theil
}

\begin{abstract}
The Extended Kalman Filter is used extensively for inertial navigation. If initial attitude errors are small, many authors choose to represent the attitude states as a vector of small angles in the vehicle body frame. Some authors choose to represent this vector in the navigation frame instead, but the corresponding reduction of filter performance in the closed loop filter is not discussed. Performance is regained when switching to an open loop filter, but closed loop filters are widely desired. This paper investigates this performance reduction. To show the effect, Monte Carlo simulation results are shown for several cases with a simplified inertial navigation problem using a closed and open loop filter and attitude states in the body and inertial frames. A qualitative argument is given to explain the effects, which stem from a state propagation model that poorly reflects the true system model for this case. A method is proposed to regain performance by using an estimated inertial frame for the attitude states. This method is only beneficial when the attitude states are measured indirectly via the velocity state equation. Results with this new frame are shown and discussed.
\end{abstract}

Stephen Steffes

DLR German Aerospace Center, Institute of Space Systems, Navigation and Control Systems Department, Robert-Hooke-Str. 7, 28359 Bremen, Germany, e-mail: stephen.steffes@dlr.de

Jan Philipp Steinbach

Helmut-Schmidt-University, University of the Federal Armed Forces Hamburg, Institute of Automation Technology, Holstenhofweg 85, 22043 Hamburg, Germany e-mail: jan.steinbach@ hsuhh.de

Stephan Theil

DLR German Aerospace Center, Institute of Space Systems, Navigation and Control Systems Department, Robert-Hooke-Str. 7, 28359 Bremen, Germany, e-mail: stephan.theil@dlr.de 


\section{Introduction}

Attitude determination techniques continue to be widely researched [1]. For vehicle navigation, much of the literature defines a small angle attitude error vector (the difference between the estimated and true attitude references) if the attitude error is expected to be relatively small. This vector is often represented in the body frame, but some authors choose to use the navigation frame instead. However, a discussion of the corresponding reduction of filter performance in the closed loop filter has not been seen by the present authors and is the motivation for this research.

The choice of frame is one factor which determines the complexity of the state transition and state update equations and the performance of the filter. Other than simpler state equations, a big advantage of using an inertial navigation frame (or a slowly changing navigation frame) is seen in real time applications where the filter corrections are calculated after the reference time of the update[2]. In this case, the attitude error states are not rotated in the filter propagation step, making the attitude updates insensitive to the computational and measurement delays. In contrast, if the body frame is used then the attitude corrections must be transformed to the current body frame when correcting the attitude states.

Attitude estimates are updated as part of the EKF update routine. In this work a small angle quaternion defines the error in the estimated attitude quaternion. A small angle attitude error vector is part of this quaternion and has additive errors to first order. Crassidis, Markley and Cheng[1], Crassidis[3], Gray[2] and Markley[4] use attitude errors in the B frame, Farrell[5] and Wendel[6] use attitude errors in the North-East-Down frame, and Gai[7], Gray[2] and Thompson and Quasius[8] use an inertial frame. All of these authors simply add the EKF update corrections to the attitude error estimates, which does not change the frame of the attitude error vector. If the update does change the frame of the attitude error vector then the attitude covariance states much be rotated to the new frame.

This paper investigates the use of a small angle attitude error vector in an inertial frame for an INS using the closed loop EKF. The closed and open loop EKFs are first presented as background for the discussion. A simple INS problem is then described where a small angle attitude error vector can be represented in either the inertial or body frames. System models, measurement models, state vectors and state transition equations are given for both attitude error representations. Simulation results are then given, which show the performance of using the closed and open loop EKFs in combination with both attitude error representations. The degraded performance of the closed loop EKF with attitude errors in the inertial frame is further analyzed. A new estimated inertial frame is proposed for the attitude error vector to improve performance of this case. Simulation results are given for this new case showing improved performance and are further discussed. 


\section{Extended Kalman Filter}

The EKF is discussed in numerous references $[9,10,11,5,12,6]$ and is summarized here. The EKF can be used in a closed-loop or open-loop manner[12]. The equations for both methods are listed in Table 1 . The notation $t_{k^{-}}$is used to denote the time immediately before the updates and $t_{k^{+}}$denotes the time immediately after. In the closed-loop method, corrections to the state estimates are fed back to the current estimated state vector $(\hat{\mathbf{x}})$ during the update routine. The estimated state vector always represents the most accurate estimate of the states, which allows the most accurate calculation of the state estimate propagation. In the open-loop method, corrections to the state estimates are not fed back to the estimated state vector but are instead added to the current estimated error state vector $(\delta \hat{\mathbf{x}})$. The estimated state vector is corrected with the estimated error state vector to get the most accurate state estimates. This type of filter is commonly used when the state propagation calculations cannot be changed directly. For additive errors the error state vector is defined as:

$$
\delta \mathbf{x}(t) \equiv \mathbf{x}(t)-\hat{\mathbf{x}}(t)
$$

where $\mathbf{x}$ is the true (error free) state vector. Note that the Error State Propagation and Whole State Update equations in Table 1 all assume the errors are additive.

Table 1 Summary of continuous-discreet open-loop and closed-loop EKF equations [9, 12]. Equations on the left are only for the closed-loop filter, those on the right are for the open-loop filter, and the equations in the middle are for both types of filters.

\begin{tabular}{|c|c|}
\hline & Closed-Loop \\
\hline System Model & $\dot{\mathbf{x}}(t)=f(\mathbf{x}(t), t)+\mathbf{w}(t) ; \mathbf{w}(t) \sim \mathscr{N}(\mathbf{0}, Q(t))$ \\
\hline Measurement Model & $\mathbf{z}_{k}=h_{k}\left(\mathbf{x}\left(t_{k^{-}}\right)\right)+v_{k} ; k=1,2, \ldots ; v_{k} \sim \mathscr{N}\left(\mathbf{0}, R_{k}\right)$ \\
\hline Initial Conditions & $\mathbf{x}(0) \sim \mathscr{N}(\hat{\mathbf{x}}(0), P(0)) ; \delta \hat{\mathbf{x}}(0)=\mathbf{0}$ \\
\hline Other Assumptions & $E\left[\mathbf{w}(t) \mathbf{v}_{k}^{T}\right]=0 ; \forall k, \forall t$ \\
\hline State Propagation & $\dot{\hat{\mathbf{x}}}(t)=f(\hat{\mathbf{x}}(t), t)$ \\
\hline Error State Propagation & $\delta \hat{\mathbf{x}}(t)=f(\mathbf{x}(t), t)-f(\hat{\mathbf{x}}(t), t)$ \\
\hline Covariance Propagation & $\dot{P}(t)=F(\hat{\mathbf{x}}(t), t) P(t)+P(t) F(\hat{\mathbf{x}}(t), t)^{T}+Q(t)$ \\
\hline Whole State Update & $\hat{\mathbf{x}}\left(t_{k^{+}}\right)=\hat{\mathbf{x}}\left(t_{k^{-}}\right)+K_{k}\left(\mathbf{z}_{k}-h_{k}\left(\hat{\mathbf{x}}\left(t_{k^{-}}\right)\right)\right)$ \\
\hline $\begin{array}{l}\text { Error State Update } \\
\text { Covariance Update }\end{array}$ & $\begin{array}{l}\delta \hat{\mathbf{x}}\left(t_{k^{+}}\right)=\delta \hat{\mathbf{x}}\left(t_{k^{-}}\right)+K_{k}\left[\mathbf{z}_{k}-h_{k}\left(\hat{\mathbf{x}}\left(t_{k^{-}}\right)+\delta \hat{\mathbf{x}}\left(t_{k^{-}}\right)\right)\right] \\
P\left(t_{k^{+}}\right)=\left[I-K_{k} H_{k}\right] P\left(t_{k^{-}}\right)\end{array}$ \\
\hline Kalman Gain Matrix & $K_{k}=P\left(t_{k^{-}}\right) H_{k}^{T}\left[H_{k} P\left(t_{k^{-}}\right) H_{k}^{T}+R_{k}\right]^{-1}$ \\
\hline Definitions & $\begin{array}{c}F(\hat{\mathbf{x}}(t), t)=[\partial f(\mathbf{x}(t), t) / \partial \mathbf{x}(t)]_{\mathbf{X}(t)=\hat{\mathbf{x}}(t)} \\
H_{k} \equiv H_{k}\left(\hat{\mathbf{x}}\left(t_{k^{-}}\right)\right)=\left[\partial h_{k}\left(\mathbf{x}\left(t_{k}\right)\right) / \partial \mathbf{x}\left(t_{k}\right)\right]_{\mathbf{X}\left(t_{k}\right)=\hat{\mathbf{x}}\left(t_{k^{-}}\right)}\end{array}$ \\
\hline
\end{tabular}




\section{Simplified Navigation Problem}

The following simplified navigation problem will be used in simulations to compare the closed and open EKF performance using an attitude error state vector in the body and an inertial frame. Most INS applications are much more complex than this example and the performance differences of the frame and filter type choices may be hidden by other effects. The presented navigation problem is as simple as possible to highlight the desired effects.

Consider a vehicle at an Earth fixed position with an IMU providing $100 \mathrm{~Hz}$ measurements of vehicle specific force and angular velocity in the IMU body frame $(B)$ with no errors. The $B$ frame is the rectangular coordinate system of the IMU measurements. The navigator provides the position, velocity and attitude of the vehicle in an inertial navigation frame $(I)$. The $I$ frame is defined to be equal to the Earth Centered Earth Fixed (ECEF) frame at time $t=0$. The ECEF frame is centered at the Earth's center of mass with the x-axis extending through the point $\left(0^{\circ}\right.$ latitude, $0^{\circ}$ longitude), the $\mathrm{z}$-axis extending through the spin axis and the $\mathrm{y}$-axis completing the right-handed coordinate system. The ECEF frame rotates about the $\mathrm{z}$-axis relative to inertial at Earth rate $\left(\omega_{\text {Earth }}=7.2921159 e-5 \mathrm{rad} / \mathrm{s}\right)$. The Earth rotation vector in the I and ECEF frame is:

$$
\Omega^{I}=\Omega^{E C E F}=\left[0,0, \omega_{E a r t h}\right]^{T}
$$

Note that in the following sections the position, velocity, attitude, acceleration and rotation variables are a function of time. However, the $(\mathrm{t})$ time dependency notion is dropped to simplify the notation. This notion is only used to indicate discrete time points or for clarification.

The navigation algorithm calculates the vehicle state over time. The system model is given as [13, 14] (see Shuster[15] for quaternion algebra definitions):

$$
\begin{aligned}
\dot{\mathbf{r}}^{I} & =\mathbf{v}^{I} \\
\dot{\mathbf{v}}^{I} & =T\left(\mathbf{q}_{B}^{I}\right) \mathbf{a}^{B}+\mathbf{g}^{I}\left(\mathbf{r}^{I}\right) \\
\dot{\mathbf{q}}_{B}^{I} & =\frac{1}{2} \mathbf{q}_{B}^{I} \otimes\left[\begin{array}{c}
\omega_{I B}^{B} \\
0
\end{array}\right]
\end{aligned}
$$

where $\mathbf{r}^{I}$ is inertial position, $\mathbf{v}^{I}$ is inertial velocity, $\mathbf{q}_{B}^{I}$ is the $B$ to $I$ frame quaternion and $T\left(\mathbf{q}_{B}^{I}\right)$ is the equivalent transformation matrix, $\mathbf{a}^{B}$ is the measured vehicle specific force, and $\omega_{I B}^{B}$ is the measured vehicle angular velocity vector with respect to $I$ in the $B$ frame. $\otimes$ is the quaternion multiplication operator and quaternions are represented as a column vector with the scalar element last. For this example the process noise $\mathbf{w}(t)$ is zero for all time. $\mathbf{g}^{I}$ is spherical gravity in the $I$ frame and is calculated using:

$$
\mathbf{g}^{I}\left(\mathbf{r}^{I}\right)=-\mu \mathbf{r}^{I} /\left\|\mathbf{r}^{I}\right\|^{3}
$$

where $\mu=398600.4418 \mathrm{~km}^{3} / \mathrm{s}^{2}$ is the standard gravitational parameter for Earth. The IMU measures the dynamics of the vehicle, which is at a fixed position relative 
to Earth. Therefore, the acceleration and rotation are constant in the $B$ frame and:

$$
\begin{aligned}
\mathbf{a}^{B}=\mathbf{a}^{B}(0) & =T\left(\mathbf{q}_{I}^{B}(0)\right)\left(\Omega^{I} \times \Omega^{I} \times \mathbf{r}^{I}(0)-\mathbf{g}^{I}\left(\mathbf{r}^{I}(0)\right)\right) \\
\omega_{I B}^{B}=\omega_{I B}^{B}(0) & =T\left(\mathbf{q}_{I}^{B}(0)\right) \Omega^{I}
\end{aligned}
$$

The error states for $\mathbf{r}^{I}$ and $\mathbf{v}^{I}$ are additive and follow from eq. (1), but the error state for the attitude quaternion is multiplicative. To avoid the complications with using a quaternion in the state vector [1] a new quantity $\theta$ will be defined, which is a vector of small angles representing the attitude error and is approximately additive. $\theta$ will be represented later in both $B$ and $I$ frames, but until then the frame will be kept general. Consider two general reference frames $A 1$ and $A 2$. The error quaternion in the $A 1$ frame can be defined as:

$$
\mathbf{p}^{A 1} \equiv \mathbf{q}_{A 2}^{A 1} \otimes \hat{\mathbf{q}}_{A 1}^{A 2}
$$

For small angular errors:

$$
\mathbf{p}^{A 1} \approx\left[-\theta^{A 1} / 2,1\right]^{T}
$$

where $\theta^{A 1}$ is a small angle rotation vector in the $A 1$ frame. With this definition, the state vector is written as:

$$
\mathbf{x} \equiv\left[\mathbf{r}^{I}, \mathbf{v}^{I}, \theta^{A 1}\right]^{T}
$$

and the entire error state vector $\delta \mathbf{x}$ is additive.

To derive the system model for $\theta^{A 1}$, take the derivative of eq. (9):

$$
\dot{\mathbf{p}}^{A 1}=\dot{\mathbf{q}}_{A 2}^{A 1} \otimes \hat{\mathbf{q}}_{A 1}^{A 2}+\mathbf{q}_{A 2}^{A 1} \otimes \dot{\hat{\mathbf{q}}}_{A 1}^{A 2}
$$

From Savage [13]:

$$
\dot{\mathbf{q}}_{A 2}^{A 1}=\frac{1}{2} \mathbf{q}_{A 2}^{A 1} \otimes\left[\omega_{I A 2}^{A 2}, 0\right]^{T}-\frac{1}{2}\left[\omega_{I A 1}^{A 1}, 0\right]^{T} \otimes \mathbf{q}_{A 2}^{A 1}
$$

Substituting this into eq. (12) and using eq. (9) and eq. (10) gives:

$$
\begin{aligned}
{\left[\begin{array}{c}
-\frac{1}{2} \dot{\theta}^{A 1} \\
0
\end{array}\right] \approx } & \frac{1}{2} \mathbf{q}_{A 2}^{A 1} \otimes\left[\begin{array}{c}
\omega_{I A 2}^{A 2} \\
0
\end{array}\right] \otimes \hat{\mathbf{q}}_{A 1}^{A 2}-\frac{1}{2}\left[\begin{array}{c}
\omega_{I A 1}^{A 1} \\
0
\end{array}\right] \otimes \mathbf{q}_{A 2}^{A 1} \otimes \hat{\mathbf{q}}_{A 1}^{A 2} \\
& +\frac{1}{2} \mathbf{q}_{A 2}^{A 1} \otimes \hat{\mathbf{q}}_{A 1}^{A 2} \otimes\left[\begin{array}{c}
\omega_{I A 1}^{A 1} \\
0
\end{array}\right]-\frac{1}{2} \mathbf{q}_{A 2}^{A 1} \otimes\left[\begin{array}{c}
\omega_{I A 2}^{A 2} \\
0
\end{array}\right] \otimes \hat{\mathbf{q}}_{A 1}^{A 2} \\
{\left[\begin{array}{c}
\dot{\theta}^{A 1} \\
0
\end{array}\right] \approx } & {\left[\begin{array}{c}
\omega_{I A 1}^{A 1} \\
0
\end{array}\right] \otimes \mathbf{p}^{A 1}-\mathbf{p}^{A 1} \otimes\left[\begin{array}{c}
\omega_{I A 1}^{A 1} \\
0
\end{array}\right] } \\
= & {\left[\begin{array}{c}
\omega_{I A 1}^{A 1}-\frac{1}{2} \omega_{I A 1}^{A 1} \times \theta^{A 1}-\omega_{I A 1}^{A 1}+\frac{1}{2} \theta^{A 1} \times \omega_{I A 1}^{A 1} \\
\frac{1}{2} \omega_{I 1}^{A 1} \cdot \theta^{A 1}-\frac{1}{2} \theta^{A 1} \cdot \omega_{I A 1}^{A 1}
\end{array}\right] } \\
= & {\left[\begin{array}{c}
-\omega_{I A 1}^{A 1} \times \theta^{A 1} \\
0
\end{array}\right] }
\end{aligned}
$$

Taking the vector part of this equation and substituting in the $I$ and $B$ frames for $A 1$ yields the two system models: 


$$
\begin{gathered}
A 1=I: \dot{\theta}^{I} \approx 0_{3 \times 1} \\
A 1=B: \dot{\theta}^{B} \approx-\omega_{I B}^{B} \times \theta^{B}
\end{gathered}
$$

To derive $F$ the system model must first be explicitly stated in terms of the states. The equation for $\dot{\mathbf{v}}^{I}$ must be stated in terms of $\theta$. If $A 1=I$ then, using the Taylor series expansion for a small angle rotation matrix[16] and eq. (9), eq. (4) becomes:

$$
\begin{aligned}
A 1=I: \dot{\mathbf{v}}^{I} & =T\left(\mathbf{p}^{I}\right) \mathbf{a}^{\hat{I}}+\mathbf{g}^{I}\left(\mathbf{r}^{I}\right) \\
& \approx\left(I_{3 \times 3}-\left(\theta^{I} \times\right)+\frac{1}{2}\left(\theta^{I} \times\right)\left(\theta^{I} \times\right)-\ldots\right) \mathbf{a}^{\hat{I}}+\mathbf{g}^{I}\left(\mathbf{r}^{I}\right)
\end{aligned}
$$

where:

$$
\mathbf{a}^{\hat{I}} \equiv T\left(\hat{\mathbf{q}}_{B}^{I}\right) \mathbf{a}^{B}
$$

Note that only the frame of $\mathbf{a}$ is affected by the rotation, not the length of the vector. One can think of $\mathbf{a}^{\hat{I}}$ as the acceleration in an estimated $I$ frame called $\hat{I}$. Finally, if $A 1=B$ then the velocity system equation instead becomes:

$$
\begin{aligned}
A 1=B: \dot{\mathbf{v}}^{I} & =T\left(\hat{\mathbf{q}}_{B}^{I}\right) T\left(\mathbf{p}^{B}\right)^{T} \mathbf{a}^{B}+\mathbf{g}^{I}\left(\mathbf{r}^{I}\right) \\
& \approx T\left(\hat{\mathbf{q}}_{B}^{I}\right)\left(I_{3 \times 3}+\left(\theta^{B} \times\right)-\frac{1}{2}\left(\theta^{B} \times\right)\left(\theta^{B} \times\right)+\ldots\right) \mathbf{a}^{B}+\mathbf{g}^{I}\left(\mathbf{r}^{I}\right)
\end{aligned}
$$

If $A 1=I$ then the system model for the entire state vector $f(\mathbf{x}(t), t)$ consists of eq. (3), eq. (15) and eq. (17). If $A 1=B$ then it consists of eq. (3), eq. (16) and eq. (19). Calculating the Jacobian gives:

$$
F(\hat{\mathbf{x}}(t), t)=\left[\begin{array}{cccc}
0_{3 \times 3} & I_{3 \times 3} & 0_{3 \times 3} \\
-\mu /\left\|\hat{\mathbf{r}}^{I}\right\|^{3} I_{3 \times 3} & 0_{3 \times 3} & F_{v \theta} \\
0_{3 \times 3} & 0_{3 \times 3} & F_{\theta \theta}
\end{array}\right]
$$

where $0_{3 \times 3}$ is a $3 \times 3$ matrix of 0 's, $I_{3 \times 3}$ is the $3 \times 3$ identity matrix, and the gravity term is a first order approximation[5]. For $A 1=I$ the values for $F_{v \theta}$ and $F_{\theta \theta}$ are:

$$
\begin{aligned}
A 1=I: F_{v \theta} & \approx\left(I_{3 \times 3}-\frac{1}{2}(\hat{\theta} \times)\right)\left(\mathbf{a}^{\hat{I}} \times\right)+\frac{1}{2}\left(\left(\mathbf{a}^{\hat{I}} \times \hat{\theta}\right) \times\right)+\ldots \\
& =\left(\mathbf{a}^{\hat{I}} \times\right)=\left(\left(T\left(\hat{\mathbf{q}}_{B}^{I}\right) \mathbf{a}^{B}\right) \times\right) \\
A 1=I: F_{\theta \theta} & =0_{3 \times 3}
\end{aligned}
$$

and for $A 1=B$ they are:

$$
\begin{aligned}
A 1=B: F_{v \theta} & \approx-T\left(\hat{\mathbf{q}}_{B}^{I}\right)\left[\left(I_{3 \times 3}-\frac{1}{2}(\hat{\theta} \times)\right)\left(\mathbf{a}^{B} \times\right)-\frac{1}{2}\left(\left(\mathbf{a}^{B} \times \hat{\theta}\right) \times\right)+\ldots\right] \\
& =-T\left(\hat{\mathbf{q}}_{B}^{I}\right)\left(\mathbf{a}^{B} \times\right) \\
A 1=B: F_{\theta \theta} & =-\left(\omega_{I B}^{B} \times\right)
\end{aligned}
$$

where the fact that $\hat{\theta}^{A 1}=0_{3 \times 1}$ is used to reduce the $F_{v \theta}$ equations.

It is known that the vehicle position is fixed relative to Earth, which is used as a measurement to update the filter. The velocity in the ECEF frame is then zero, 
which can be calculated in terms of the inertial states by subtracting Earth rotation velocity from the inertial velocity in the ECEF frame [5]. The measurement model is then:

$$
\mathbf{z}_{k}=T_{I}^{E C E F}\left(t_{k}\right) \mathbf{v}^{I}\left(t_{k^{-}}\right)-\left(T_{I}^{E C E F}\left(t_{k}\right) \mathbf{r}^{I}\left(t_{k^{-}}\right)\right) \times \Omega^{E C E F}+v_{k}
$$

where $v_{k} \sim \mathscr{N}\left(\mathbf{0}, R_{k}\right)$ and $T_{I}^{E C E F_{k}}$ is the $I$ to $E C E F$ transformation defined as:

$$
T_{I}^{E C E F}\left(t_{k}\right)=\left[\begin{array}{ccc}
\cos \left(t_{k} * \omega_{\text {Earth }}\right) & \sin \left(t_{k} * \omega_{\text {Earth }}\right) & 0 \\
-\sin \left(t_{k} * \omega_{\text {Earth }}\right) & \cos \left(t_{k} * \omega_{\text {Earth }}\right) & 0 \\
0 & 0 & 1
\end{array}\right]
$$

Calculating the Jacobian gives:

$$
H_{k}=\left[\left(\Omega^{E C E F} \times\right) T_{I}^{E C E F}\left(t_{k}\right) T_{I}^{E C E F}\left(t_{k}\right) 0_{3 \times 3}\right]
$$

To keep the small angle approximation in eq. (10) as accurate as possible, the estimated value of $\theta^{A 1}$ will be fed back to the whole state attitude estimate after each EKF update in the closed loop case. The notation $t_{k^{+}}$will be used to denote the time after the update and before the feedback, and $t_{k^{++}}$will denote the time immediately after the feedback. Therefore, immediately after the whole state update equation the following operations are done with the estimated states:

$$
\begin{aligned}
& \hat{\mathbf{q}}_{A 2}^{A 1}\left(t_{k^{++}}\right)=\left[-\hat{\theta}^{A 1}\left(t_{k^{+}}\right) / 2,1\right]^{T} \otimes \hat{\mathbf{q}}_{A 2}^{A 1}\left(t_{k^{+}}\right) \\
& \hat{\theta}^{A 1}\left(t_{k^{+}}\right)=0_{3 \times 1}
\end{aligned}
$$

where $0_{3 \times 1}$ is a $3 \times 1$ vector of 0 's, and $\hat{\mathbf{q}}_{A 2}^{A 1}\left(t_{k^{++}}\right)$is renormalized to 1 after this operation. With this method, the estimated small angle vector $\hat{\theta}^{A 1}$ is always $0_{3 \times 1}$ during propagation. A similar procedure is used by a number of authors [3, 4] and is often called a "reset" of the attitude states, however without the explicit notation used here. The effect this has on the true state $\theta^{A 1}$ is found by starting with eq. (9) at $t_{k^{++}}$and using eq. (10), eq. (30) and the fact that $\mathbf{q}_{A 2}^{A 1}\left(t_{k^{++}}\right)=\mathbf{q}_{A 2}^{A 1}\left(t_{k^{+}}\right)$(since the true attitude is not changed by the feedback) to get:

$$
\begin{aligned}
\mathbf{p}^{A 1}\left(t_{k^{++}}\right) & =\mathbf{q}_{A 2}^{A 1}\left(t_{k^{++}}\right) \otimes \hat{\mathbf{q}}_{A 1}^{A 2}\left(t_{k^{++}}\right) \\
& =\mathbf{q}_{A 2}^{A 1}\left(t_{k^{+}}\right) \otimes \hat{\mathbf{q}}_{A 1}^{A 2}\left(t_{k^{+}}\right) \otimes\left[\frac{1}{2} \hat{\theta}^{A 1}\left(t_{k^{+}}\right), 1\right]^{T} \\
& \approx\left[-\frac{1}{2} \theta^{A 1}\left(t_{k^{+}}\right), 1\right]^{T} \otimes\left[\frac{1}{2} \hat{\theta}^{A 1}\left(t_{k^{+}}\right), 1\right]^{T} \\
{\left[-\frac{1}{2} \theta^{A 1}\left(t_{k^{++}}\right), 1\right]^{T} } & \approx\left[-\frac{1}{2} \theta^{A 1}\left(t_{k^{+}}\right)+\frac{1}{2} \hat{\theta}^{A 1}\left(t_{k^{+}}\right), 1\right]^{T} \\
\theta^{A 1}\left(t_{k^{+}}\right) & \approx \theta^{A 1}\left(t_{k^{+}}\right)-\hat{\theta}^{A 1}\left(t_{k^{+}}\right)
\end{aligned}
$$

which agrees with earlier statements that $\theta$ is approximately additive. There is no change in the covariance matrix due to these operations since this merely moves information from one place to another and does not change the statistics associated with these states[4]. Which means:

$$
P\left(t_{k^{++}}\right)=P\left(t_{k^{+}}\right)
$$




\section{Baseline Simulation Results}

Four Monte Carlo simulations are used to give a quantitative measure of the performance reduction when using the closed loop EKF with an attitude error vector in the $I$ frame. Using the closed and open loop EKF algorithms summarized in section 2, four separate simulations were run on the navigation problem discussed in section 3: closed loop with $A 1=B$ (CLB case), open loop with $A 1=B$ (OLB case), closed loop with $A 1=I$ (CLI case), and open loop with $A 1=I$ (OLI case).

For every case, the simulation was setup as follows. The filter starts at $t=0$ and ends at $t=10 . \mathbf{r}^{I}(0)=[6378137 m, 0,0]^{T}$, which is at $0^{\circ}$ latitude, $0^{\circ}$ longitude on Earth's surface. $\mathbf{v}^{I}(0)$ is set to the Earth surface velocity, which is $\Omega^{E C E F} \times \mathbf{r}^{I}(0)$. $\mathbf{q}_{B}^{I}(0)$ is set to a random quaternion $[\hat{e} \sin (\alpha / 2), \cos (\alpha / 2)]^{T}$ with $\alpha \sim \mathscr{U}\left(0,360^{\circ}\right)$ and $\hat{e}$ uniformly random over the unit sphere. The estimated states are set to $\hat{\mathbf{r}}^{I}(0)=\mathbf{r}(0)+v_{r}, \hat{\mathbf{v}}^{I}(0)=\mathbf{v}(0)+v_{v}, \hat{\theta}^{A 1}(0)=[0,0,0]^{T}$, and $\hat{\mathbf{q}}_{B}^{I}(0)=\mathbf{p} / \otimes \mathbf{q}_{B}^{I}$, where $v_{r} \sim \mathscr{N}\left(0, \sigma_{r} I_{3 \times 3}\right), v_{v} \sim \mathscr{N}\left(0, \sigma_{v} I_{3 \times 3}\right), \mathbf{p}$ ' is the quaternion $\left[q_{1}, q_{2}, q_{3}, q_{4}\right]^{T}$ with $q_{1}, q_{2}, q_{3} \sim \mathscr{N}\left(0, \sigma_{\theta}^{2}\right)$ and $q_{4}=\sqrt{q_{1}^{2}+q_{2}^{2}+q_{3}^{2}} . P(0)$ is set to:

$$
P(0)=\left[\begin{array}{ccc}
\sigma_{r}^{2} I_{3 \times 3} & 0_{3 \times 3} & 0_{3 \times 3} \\
0_{3 \times 3} & \sigma_{v}^{2} I_{3 \times 3} & 0_{3 \times 3} \\
0_{3 \times 3} & 0_{3 \times 3} & \sigma_{\theta}^{2} I_{3 \times 3}
\end{array}\right]
$$

where $\sigma_{r}=1 \mathrm{~m}, \sigma_{v}=0.1 \mathrm{~m} / \mathrm{s}$, and $\sigma_{\theta}=0.07 \mathrm{rad}\left(=4^{\circ}\right)$. As previously mentioned, there is no system noise, so $\mathbf{w}(t)$ is 0 at all time. There is also no measurement noise since it is known that the ECEF velocity is exactly zero, but using zero measurement noise can lead to division by zero in the Kalman gain matrix. Therefore, $R_{k}$ is set to $(0.001 \mathrm{~m} / \mathrm{s})^{2} I_{3 \times 3}$. The state propagation equations are integrated at $100 \mathrm{~Hz}$ using forward Euler for eq. (3), forward Euler with rotation correction[14] for eq. (4), a 3rd order quaternion integration method[16] for eq. (5) and the exact solutions for eq. (15) and eq. (16).

100 simulations were run for each Monte Carlo and the same set of initial conditions are used for each Monte Carlo. Figure 1 shows the results. For each case at each time point, the root mean square (RMS) of the attitude errors and the corresponding $1 \sigma$ values for all 100 simulations are plotted. The position and velocity errors are all near or below their RMS $1 \sigma$ values and are not shown.

\section{Analysis of Simulation Results}

The filter performances for the CLB, OLI, and OLB cases are all as expected; the RMS errors are all near or below the $1 \sigma_{R M S}$ values. However, the performance of the CLI case is very poor. After the second measurement update (at $2 \mathrm{sec}$ ) the error in attitude is well outside of the $1 \sigma_{R M S}$ boundary. The fact that both open loop cases perform as expected suggests that the poor performance of the CLI case is related 


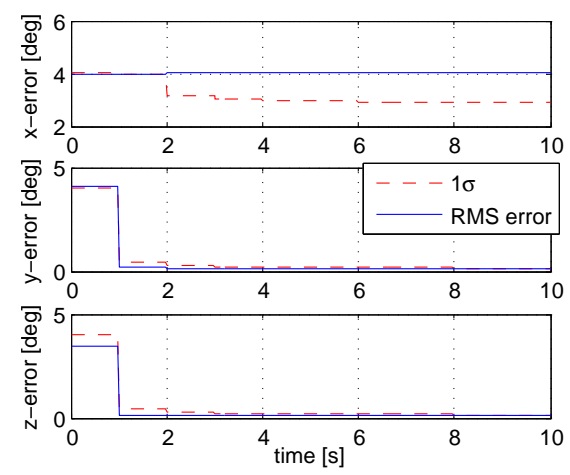

(a)
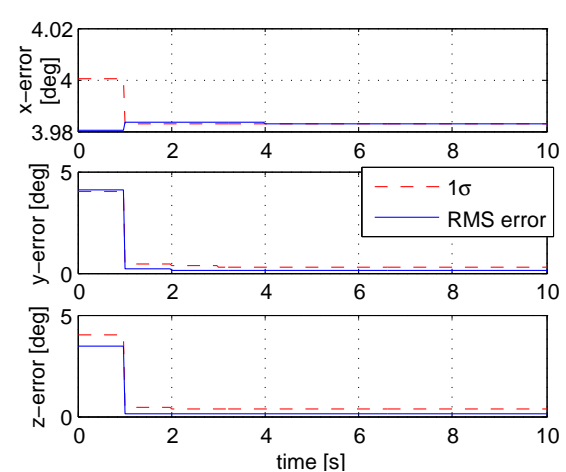

(c)
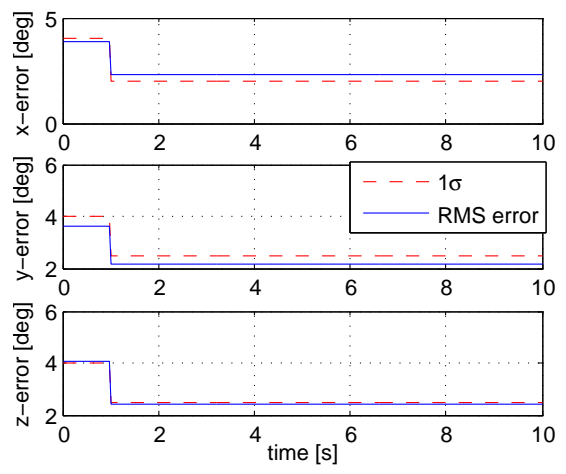

(b)
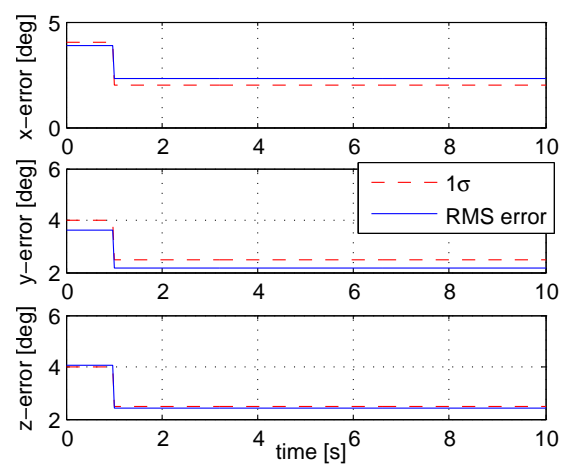

(d)

Fig. 1 RMS attitude error CLI (a), CLB (b), OLI (c) and OLB (d) Monte Carlo simulations.

to feeding back the state corrections in the EKF update routine. The fact that both cases with $A 1=B$ perform as expected suggests that the CLI performance is also related to the state transition equations. Both the velocity and attitude propagation terms depend on the choice of $A 1$, but the attitude terms (eq. (23) and eq. (26)) can be eliminated as suspect since they do not depend on the state corrections. The velocity terms (eq. (22) and eq. (25)) contain $\hat{\mathbf{q}}_{B}^{I}$, which is corrected after every EKF update with eq. (30). It seems that the velocity terms are causing the CLI performance problem since the attitude corrections come from updates with velocity measurements. The following qualitative approach explains why the CLI case inherently has poor performance in these simulations.

In eq. (22), $\mathbf{a}^{B}$ is first transformed with the attitude estimate into the $\hat{I}$ frame and this is used in a cross product matrix which is multiplied with the covariances of $\theta^{I}$ during the EKF propagation ${ }^{1}$. When the attitude estimate is corrected from the EKF

${ }^{1}$ Additionally, note that crossing two vectors in different frames yields a resultant vector in an undefined frame. 
update the direction of $\mathbf{a}^{\hat{I}}$ changes at this instant, even though the direction of $\mathbf{a}^{I}$ did not. In effect, the $\hat{I}$ frame changes even though the true attitude does not change at this instant. This is misinterpreted by the EKF as a change in the acceleration direction relative to $\theta^{I}$, which causes poor filter performance since this does not reflect the system model.

In contrast, in eq. (25), $\mathbf{a}^{B}$ is first crossed with the attitude state covariances and then this cross product is transformed with the attitude estimate. Again the attitude estimate is changed by the update, but the relationship between $\mathbf{a}^{B}$ and the attitude covariance (i.e. the cross product matrix) is not affected. The EKF does not interpret the attitude update as a change in acceleration, but it does misinterpret it as a change in the entire cross product. Clearly, the EKF is not sensitive to this misinterpretation since the performance of the CLB case is OK.

Finally, in the OLI case the attitude corrections are accumulated in the error state vector instead of updating the attitude estimate. This eliminates the false acceleration direction changes in the CLI case, therefore providing better performance.

The Monte Carlo simulations used a relatively large initial attitude error of $\sigma_{\theta}=$ $4^{\circ}$. As this error is reduced, the size of the attitude updates is reduced and the CLI case shows increasingly better performance. For the presented simulation setup, an initial attitude error of $\sigma_{\theta} \approx 0.05^{\circ}$ was found to be the boundary between good and poor performance for the CLI case, where "good" performance means the RMS attitude and velocity errors are near or below their respective $1 \sigma_{R M S}$ boundaries.

\section{Closed Loop with $A 1=\hat{I}$ Case}

The cross product in eq. (22) crosses a vector in the $\hat{I}$ frame with the covariances of $\theta$ in the $I$ frame. Crossing vectors in two different frames in general does not make sense because the frame of the product is not defined. A better approach might be to use some $\theta^{\hat{I}}$ as the attitude state instead. To show how this simple, yet unconventional change affects the EKF performance, the system model and update equations will be derived and Monte Carlo results for the resulting system will be shown.

To start, $\theta^{\hat{I}}$ is first defined as:

$$
\theta^{\hat{I}} \equiv T\left(\hat{\mathbf{q}}_{B}^{I}\right) T\left(\mathbf{q}_{I}^{B}\right) \theta^{I}
$$

Using eq. (9) this becomes:

$$
\theta^{\hat{I}}=T\left(\left(\mathbf{p}^{I}\right)^{-1}\right) \theta^{I}
$$

This can be further reduced using a Taylor series expansion to:

$$
\begin{aligned}
& \theta^{\hat{I}} \approx\left(I_{3 x 3}+\left(\theta^{I} \times\right)-\frac{1}{2}\left(\theta^{I} \times\right)\left(\theta^{I} \times\right)+\ldots\right) \theta^{I} \\
& \theta^{\hat{I}} \approx \theta^{I}
\end{aligned}
$$


It is already known that $\theta^{I}$ (and thus $\mathbf{p}^{I}$ ) are constant during the EKF propagation from eq. (15). Therefore, the system model for this state must be:

$$
\dot{\theta}^{\hat{I}}=0_{3 \times 1}
$$

To find the system model for velocity combine eq. (17) and eq. (38) to get:

$$
\dot{\mathbf{v}}^{I} \approx\left(I_{3 \times 3}-\left(\theta^{\hat{I}} \times\right)+\frac{1}{2}\left(\theta^{\hat{I}} \times\right)\left(\theta^{\hat{I}} \times\right)-\ldots\right) \mathbf{a}^{\hat{I}}+\mathbf{g}^{I}\left(\mathbf{r}^{I}\right)
$$

Therefore, the $F$ matrix for this case is the same as in the CLI case. Additionally, the measurement equations are unchanged since they are not affected by the choice of $A 1$.

To keep small angle approximations as accurate as possible, the estimated value of $\theta^{\hat{I}}$ will be fed back to the whole state attitude estimate after each EKF update, analogous to the procedure in eq. (30) and eq. (31). Immediately after the whole state update equation the following operations are done:

$$
\begin{aligned}
\hat{\mathbf{q}}_{B}^{I}\left(t_{k^{++}}\right) & =\left[-\hat{\boldsymbol{\theta}}^{\hat{I}}\left(t_{k^{+}}\right) / 2,1\right]^{T} \otimes \hat{\mathbf{q}}_{B}^{I}\left(t_{k^{+}}\right) \\
\hat{\theta}^{\hat{I}}\left(t_{k^{++}}\right) & =0_{3 \times 1}
\end{aligned}
$$

As a result, the $\hat{I}$ frame changes discretely at the update times. The resulting change in $\theta^{\hat{I}}$ is calculated by starting with eq. (36) at $t_{k^{+}}$and using parts of eq. (32) and eq. (36) again to get:

$$
\begin{aligned}
\theta^{\hat{I}}\left(t_{k^{++}}\right) & =T\left(\left(\mathbf{p}^{I}\left(t_{k^{++}}\right)\right)^{-1}\right) \theta^{I}\left(t_{k^{++}}\right) \\
& \approx T\left(\left[\frac{1}{2} \theta^{I}\left(t_{k^{++}}\right), 1\right]^{T}\right) \theta^{I}\left(t_{k^{++}}\right) \\
& \approx T\left(\left[-\frac{1}{2} \hat{\theta}^{I}\left(t_{k^{+}}\right), 1\right]^{T} \otimes\left[\frac{1}{2} \theta^{I}\left(t_{k^{+}}\right), 1\right]^{T}\right)\left(\theta^{I}\left(t_{k^{+}}\right)-\hat{\theta}^{I}\left(t_{k^{+}}\right)\right) \\
& \approx T\left(\left[-\frac{1}{2} \hat{\theta}^{I}\left(t_{k^{+}}\right), 1\right]^{T}\right)\left(T\left(\left[\frac{1}{2} \theta^{I}\left(t_{k^{+}}\right), 1\right]^{T}\right) \theta^{I}\left(t_{k^{+}}\right)-T\left(\left[\frac{1}{2} \theta^{I}\left(t_{k^{+}}\right), 1\right]^{T}\right) \hat{\theta}^{I}\left(t_{k^{+}}\right)\right) \\
& \approx T\left(\left[-\frac{1}{2} T\left(\mathbf{p}^{I}\right) \hat{\theta}^{\hat{I}}\left(t_{k^{+}}\right), 1\right]^{T}\right)\left(\theta^{\hat{I}}\left(t_{k^{+}}\right)-\hat{\theta}^{\hat{I}}\left(t_{k^{+}}\right)\right) \\
& \approx T\left(\left[-\frac{1}{2} \hat{\theta}^{\hat{I}}\left(t_{k^{+}}\right), 1\right]^{T}\right)\left(\theta^{\hat{I}}\left(t_{k^{+}}\right)-\hat{\theta}^{\hat{I}}\left(t_{k^{+}}\right)\right)
\end{aligned}
$$

The last equation shows that $\theta^{\hat{I}}$ is not additive because the $\hat{I}$ frame changes by the rotation $T\left(\left[-\frac{1}{2} \hat{\theta}^{\hat{I}}\left(t_{k^{+}}\right), 1\right]^{T}\right)$ due to the feed back operations. In this case the covariance matrix must also be updated to reflect the frame change. Therefore, as a final step in the update routine, a discrete propagation of the covariance matrix must be done from $t_{k^{+}}$to $t_{k^{++}}$to change the frame of the attitude covariances. The discrete form of the EKF propagation equations in Table 1 are $[9,12]$ :

$$
\begin{aligned}
\hat{\mathbf{x}}\left(t_{k^{++}}\right) & =f\left(\hat{\mathbf{x}}\left(t_{k^{+}}\right)\right) \\
P\left(t_{k^{++}}\right) & =F_{k^{+}} P\left(t_{k^{+}}\right) F_{k^{+}}^{T}+Q_{k^{+}} \\
F_{k^{+}} \equiv F\left(\hat{\mathbf{x}}\left(t_{k^{+}}\right)\right) & =[\partial f(\mathbf{x}(t)) / \partial \mathbf{x}(t)]_{\mathbf{x}(t)=\hat{\mathbf{x}}\left(t_{k^{+}}\right)}
\end{aligned}
$$

The $f$ function does not change $\mathbf{r}^{I}$ or $\mathbf{v}^{I}$, but uses eq. (43) for the attitude states. From the Jacobian of eq. (43), the system matrix is: 


$$
F_{k^{+}}=\left[\begin{array}{ccc}
I_{3 \times 3} & 0_{3 \times 3} & 0_{3 \times 3} \\
0_{3 \times 3} & I_{3 \times 3} & 0_{3 \times 3} \\
0_{3 \times 3} & 0_{3 \times 3} & T\left(\left[-\frac{1}{2} \hat{\theta}^{\hat{I}}\left(t_{k^{+}}\right), 1\right]^{T}\right)
\end{array}\right]
$$

and the process noise is $Q_{k^{+}}=\mathbf{0}$. The EKF update is finally complete with these last steps.

A Monte Carlo of 100 simulations, like those discussed in section 4, was done for the closed loop $A 1=\hat{I}$ case to give a quantitative measure its performance. 100 simulations were run and the same set of initial conditions from section 4 are used. Figure 2 show the results. As with the other simulation results, the root mean square (RMS) of the velocity errors, attitude errors, and their corresponding $1 \sigma$ values for all 100 simulations are plotted. The position errors are not shown because they are approximately an integral of the corresponding velocity errors and are therefore not interesting.

The filter performance for this case is similar to the CLB, OLI and OLB cases. The RMS errors are all near or below the $1 \sigma_{R M S}$ boundaries.

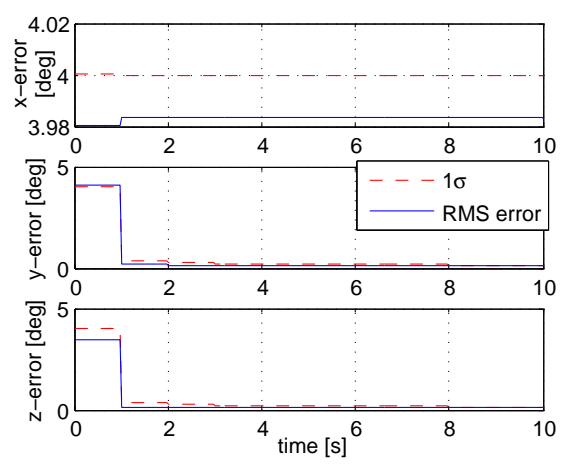

Fig. 2 RMS attitude error for closed loop EKF with $A 1=\hat{I}$ Monte Carlo.

\section{Conclusion}

The presented work has shown how the choice of the attitude error vector frame and filter type can effect filter performance. Four separate Monte Carlo simulations were done for a simplified navigation problem. The filter performance for the CLB, OLI and OLB cases was as expected, but the performance of the CLI case was relatively poor. In all cases, the attitude corrections were estimated indirectly via the velocity measurements with the $F_{v \theta}$ term in the EKF propagation equation. In the CLI case, the $\mathbf{a}^{\hat{I}}$ term in $F_{v \theta}$ changes after the EKF update but the attitude covariance 
states are unchanged. The poor performance of the CLI case can be attributed to this inconsistent modeling.

To avoid the problems with the CLI case, the $\hat{I}$ frame was used to represent the attitude states instead. This case has the same propagation equations as the CLI case, but uses an additional discrete filter propagation step immediately after the normal EKF update to rotate the attitude covariance states to the new $\hat{I}$ frame. In this case both the $\mathbf{a}^{\hat{I}}$ term in $F_{v \theta}$ and the attitude covariance states are always in the current $\hat{I}$ frame, which fixes the inconsistent modeling problem from the CLI case. Monte Carlo results for this case show similar performance to the CLB, OLI and OLB cases.

Most INS systems are much more complicated than the example used in this work. However, if a filter like the CLI case is used then the inconsistent modeling issues discussed in this work may degrade performance. For any system, all options should be considered to find the best choice for the application.

\section{References}

1. John L. Crassidis, F. L. M. and Cheng, Y., "Survey of Nonlinear Attitude Estimation Methods," Journal of Guidance, Control and Dynamics, Vol. 30, No. 1, Jan.-Feb. 2007, pp. 12-28.

2. Gray, C. W., "Star Tracker/IRU Attitude Determination Filters," Vol. 107, 2001, pp. 459-476, AAS 01-039.

3. Crassidis, J. L., "Sigma-Point Kalman Filtering for Integrated GPS and Inertial Navigation," AIAA Guidance, Navigation, and Control Conference, San Francisco, CA, August 2005, AIAA-2005-6052.

4. Markley, F. L., "Attitude Error Representations for Kalman Filtering," Journal of Guidance, Control and Dynamics, Vol. 26, No. 2, Mar.-Apr. 2003, pp. 311-317.

5. Farrell, J. A., Aided Navigation: GPS with High Rate Sensors, Mc Graw Hill, 2008.

6. Wendel, J., Integrierte Navigationssysteme: Sensordatenfusion, GPS und Inertiale Navigation, Oldenbourg Wissenschaftsverlag GmbH, 2007.

7. Eliezer Gai, Kevin Daly, J. H. and Lemos, L., "Star-Sensor-Based Satellite Attitude/Attitude Rate Estimator," Journal of Guidance, Control and Dynamics, Vol. 8, No. 5, Sep.-Oct. 1985, pp. 560-565.

8. Thompson, I. C. and Quasius, G. R., "Attitude Determination for the P80-1 Satellite," In Proceedings of AAS Guidance and Control Conference, 1980, AAS 80-001.

9. Gelb, A., editor, Applied Optimal Estimation, The MIT Press, 1974.

10. Brown, R. and Hwang, P., Introduction to Random Signals and Applied Kalman Filtering, John Wiley and Sons, 1997.

11. Grewal, M. S. and Andrews, A. P., Kalman Filtering - Theory and Practice using MATLAB, John Wiley \& Sons, Second ed., 2001.

12. Kayton, M. and Fried, W. R., Avionics Navigation Systems, John Willey and Sons Inc., 2nd ed., 1997.

13. Savage, P. G., "Strapdown Inertial Navigation Integration Algorithm Design Part 1: Attitude Algorithms," Journal of Guidance, Control, and Dynamics, Vol. 21, No. 1, 1998, pp. 19-28.

14. Savage, P. G., "Strapdown Inertial Navigation Integration Algorithm Design Part 2: Velocity and Position Algorithms," Journal of Guidance, Control, and Dynamics, Vol. 21, No. 2, 1998, pp. 208-221.

15. Shuster, M. D., "A Survey of Attitude Representations," Journal of the Astronautical Sciences, Vol. 41, No. 4, 1993, pp. 439-517. 
16. McKern, R. A., A Study of Transformation Algorithms for Use in a Digital Computer, Master's thesis, Massachusetts Institute of Technology, January 1968. 